\title{
ADJOINT TRANSFORM, OVERCONVEXITY AND SETS OF CONSTANT WIDTH
}

\author{
FRANÇOIS BAVAUD
}

\begin{abstract}
The properties of the adjoint transform (associating to a set the intersection of all disks of given radius centered in the set) are systematically investigated, in particular its relationship with the overconvex, the parallelisation and completion of sets. Sets conjugate by the transform can be characterised in a new way as the union or the intersection of all completions of the reference body. New relationships satisfied by their areas and perimeters are derived. Two applications in problems of random intersection of disks are finally treated.
\end{abstract}

\section{INTRODUCTION}

Let $K$ be a compact, plane (possibly nonconvex) set, and $s$ a positive real number. We call s-adjoint transform $\mathscr{A}(\ldots, s)$ the operation which to $K$ associates its $s$-adjoint $K^{*}(s)$, defined as the intersection of all disks of radius $s$ whose centers lie in $K$ :

$$
K^{*}(s):=\mathscr{A}(K, s):=\bigcap_{x \in K} B(x, s)
$$

where $B(x, s)$ denotes the disk of center $x$ and radius $s$.

Judging from the existing literature, it appears that different authors (including the present one) have independently made use of the operation (1) in one way or another, with different applications in mind: in the thirties, Mayer [13] and Bückner [7] explored the notion of overconvexity, the former for its own sake, the latter as a tool for the study of the sets of constant width. A decade later, Blanc $[4,5]$ investigates relations linking the overconvexity and the adjoint transform. In the sixties, Eggleston [10] characterizes the complete sets as fixed points of the adjoint transform. Let us also mention Maehara [12] for his paper on sets conjugate with respect to the adjoint transform, and Sallee $[14,15]$ for his generalised adjoint transform which associates to a set $K$ the intersection of all $K$-translates of a given centrally symmetric convex body. Our own interest has arisen initially from considerations relative to the theory of random polytopes.

We have tried to keep the level of exposition as self-contained and elementary as possible. Already known facts previously scattered in the literature are gathered here in a unified exposition. Many results appear here for the first time to your knowledge: we have attempted to dignify the most evocative among them

Received by the editors June 6, 1990.

1980 Mathematics Subject Classification (1985 Revision). Primary 52A10, 52A22. 
as theorems. We have restricted ourselves in this paper to two-dimensional, euclidean geometry. If one sees no reason forbidding the straightforward generalisation of most of our results in higher dimensions, one nevertheless knows (see e.g. [10]) that it is not the case anymore for Minkowskian geometries.

Section 2 reviews the basic properties of the transform. In $\S 3$, we generalise to areas a result of Blanc concerning the perimeters of a conjugate pair; investigating the composition of the adjoint transform with the operation of parallelisation, we obtain differential equations obeyed by the areas and perimeters of a conjugate pair, somehow analogous to the Steiner formulae for parallel bodies. We propose in $\S 4$ a construction (one more!) for completing a plane body; this leads to the new characterisation of the elements of a conjugate pair as the union, respectively the intersection of all the completions containing the reference body. In $\S 5$ we study the stochastic processes arising from the random intersection of disks of fixed radius, each containing the centers of all the other disks. The differences between the sequential and the simultaneous processes are elucidated.

\section{BASIC PROPERTIES}

We denote the area, perimeter, diameter, minimal width, circumcenter and circumradius of $K$ by $|K|,|\partial K|, d(K), \delta(K), C_{e}(K)$ and $R(K)$ respectively. $|x-y|$ stands for the euclidean distance between two points $x$ and $y$.

The following properties are immediate consequences of the definition (1):

(a) $\mathscr{A}\left(\bigcup_{i} K_{i}, s\right)=\bigcap_{i} \mathscr{A}\left(K_{i}, s\right)$,

(b) $\mathscr{A}\left(\bigcap_{i} K_{i}, s\right) \subseteq \mathscr{A}\left(\bigcup_{i} K_{i}, s\right)$,

(c) $K^{*}(s)$ is a nonempty convex set provided $s \geq R(K)$, and $K^{*}(R(K))=$ $\left\{C_{e}(K)\right\}$,

(d) $K^{*}(s) \subset K^{*}\left(s^{\prime}\right)$ iff $s<s^{\prime}$,

(e) $K^{*}(s) \subset \widetilde{K}^{*}(s)$ if $\widetilde{K} \subset K$,

(f) $K \subseteq K^{*}(s)$ iff $s \geq d(K)$.

We shall as well consider the double adjoint transform $\mathscr{A}^{2}(\ldots, s)$, defined as the operation which to $K$ associates $K^{* *}(s)$, the adjoint of its adjoint:

$$
K^{* *}(s):=\mathscr{A}^{2}(K, s):=\mathscr{A}(\mathscr{A}(K, s))=\mathscr{A}\left(K^{*}(s), s\right)=\bigcap_{x \in K^{*}(s)} B(x, s) .
$$

Let us first check that, provided $K^{*}(s)$ itself is nonempty (i.e. $\left.s \geq R\right), K^{* *}(s)$ is also nonempty; we actually have

(g) $K \subseteq K^{* *}(s)$, for all $s \geq R$.

Secondly, one more iteration of the adjoint transform does not bring anything new; using an obvious notation, one has

(h) $K^{* * *}(s)=K^{*}(s)$.

(Proofs of (g) and (h) can be found e.g., in [12 or 15].)

As $x \in K^{*}(s)$ iff $K \subseteq B(x, s)$, we get from (2) the equivalent characterisation

(i) $K^{* *}(s)=\bigcap_{B(x, s) \supseteq K} B(x, s)$.

As a consequence

(j) $K^{* *}(s) \subseteq K^{* *}\left(s^{\prime}\right)$ if $s \geq s^{\prime}$,

(k) $K^{* *}(s) \subseteq \widetilde{K}^{* *}(s)$ if $K \subseteq \widetilde{K}$.

From (i) and (j), the following picture emerges: 
(1) For $s \in[R, \infty)$, the sets $K^{* *}(s)$ constitute a decreasing nested family of convex sets, the largest one being $K^{* *}(R)$, the circumcircle of $K$, and the smallest one being $\lim _{s \rightarrow \infty} K^{* *}(s)=\bar{K}$, the convex hull of $K$. (The limit refers here to the Hausdorff metric or any equivalent metric: see e.g. [6].)

In particular, all the members of the family coincide when $K$ is a circle. Keeping in mind that $\bar{K}$ is obtained from $K$ by adjunction of all points belonging to any segment whose endpoints lie in $K$, one might wonder at this stage whether a similar construction exists for the other members of the family. The answer is positive, and leads to the very heart of the concept of overconvexity, introduced independently by Mayer [13] and Bückner [7] (the latter speaks of "sets rounded off to the index $s$ "):

Definition. A set $K$ is said to be s-overconvex if, for any pair of points $x$ and $y$ belonging to $K$, the closed lens resulting from the intersection of the two disks of radius $s$ passing through $x$ and $y$ is contained in $K$.

Clearly, a circle of radius $R$ is $s$-overconvex iff $s \geq R$, and more generally a set is $s$-overconvex iff its curvature radius satisfies $0 \leq \rho(\phi) \leq s$, for all $\phi \in[0,2 \pi]$ [13]. In the limit $s \rightarrow \infty$ one recovers the usual notion of convexity. As the intersection of two $s$-overconvex sets is still $s$-overconvex, the adjoint $K^{*}(s)$ of $K$ (and therefore $\left.K^{* *}(s)\right)$ is $s$-overconvex. From (i), $K^{* *}(s)$ is the set obtained by rounding off $K$ by arcs of radius $s$, and we get the following link between the concepts of adjoint transform and convexity [4]:

(m) $K=K^{* *}(s)$ iff $K$ is $s$-overconvex.

Incidentally, it comes from properties $(\mathrm{h})$ and $(\mathrm{m})$ that the adjoint transform, applied on sets of points, enjoys properties very similar to those associated with the Legendre-Fenchel transform (see e.g. [8]) applied on functions. In particular, we leave to the reader to verify:

(n) $K$ is $s$-overconvex iff it is the $s$-adjoint transform of some convex set.

Moreover, taking (d) into account:

(o) Any $s$-overconvex set is contained in some disk of radius $s$.

\section{Conjugacy PROPERTIES}

As a consequence of (2) and (h), $K^{*}(s)$ and $K^{* *}(s)$ are obtained from each other by means of the same operation: we propose therefore to refer to them as a conjugate pair. For fixed $s$, the larger is a set, the smaller is its conjugate: for instance, the conjugate to the circle of radius $R(R \leq s)$ is the circle of radius $s-R$. This "competition" between conjugates is quantitatively expressed in the following theorem:

Theorem I. The perimeters $\left|\partial K^{*}(s)\right|,\left|\partial K^{* *}(s)\right|$ and areas $\left|K^{*}(s)\right|,\left|K^{* *}(s)\right|$ of a conjugate pair satisfy

$$
\begin{gathered}
\left|\partial K^{*}(s)\right|+\left|\partial K^{* *}(s)\right|=2 \pi s . \\
2\left|K^{*}(s)\right|-s\left|\partial K^{*}(s)\right|=2\left|K^{* *}(s)\right|-s\left|\partial K^{* *}(s)\right| .
\end{gathered}
$$

The first relation is due to Blanc [5], whereas we believe the second to be new.

Proof. Given a set $K$, we denote by $h(\phi)$ its support function, that is $h(\phi):=$ $\sup \left\{x_{1} \cos (\phi)+x_{2} \sin (\phi) \mid x=\left(x_{1}, x_{2}\right) \in K\right\}$. By construction, the support 
functions $h^{*}(\phi)$ and $h^{* *}(\phi)$ of $K^{*}(s)$ and $K^{* *}(s)$ satisfy

$$
h^{*}(\phi)+h^{* *}(\pi+\phi)=s \text {. }
$$

(Actually, (5) can be shown to be not only a necessary condition for two support functions to describe a conjugate pair, but also a sufficient one [12].) The perimeter of a set being equal to the integral of its support function (see e.g. [6]), one immediately gets (3). On the other hand, the area of a set $K$ is given by

$$
|K|=\int_{0}^{2 \pi} \rho(\phi) h(\phi) d \phi
$$

where $\rho(\phi)=h(\phi)+h^{\prime \prime}(\phi)$ is the curvature radius. Taking into account $\rho^{*}(\phi)+$ $\rho^{* *}(\pi+\phi)=s$ as well as $(5)$, one gets

$$
\left|K^{*}(s)\right|=K^{* *}(s)|-s| \partial K^{* *}(s) \mid+\pi s^{2}
$$

which, using (3), is equivalent to (4).

Remark. An alternative proof of (4) starts with the observation that a point $x$ belongs to $K^{*}(s)$ iff $K^{* *}(s) \subseteq B(x, s)$; this implies the area of $K^{*}(s)$ to be the measure of all translations of $K^{* *}(s)$ (or $K$ ) in which it is contained in a disk of radius $s$. As the curvature radius of $K^{* *}(s)$ does not exceed $s$, the above measure can be computed explicitly (see e.g. [16, p. 95]) and coincides precisely with the right-hand side of (7).

A third proof of (4) is based upon Poincaré's formula (see e.g. [16, p. 111]). Let $n(x)$ be the number of intersections of the circle $\partial B(x, s)$ with the boundary $\partial K^{*}(s)$ of $K^{*}(s)$. Up to sets of measure zero, $n(x)=2$ for $x \in$ $\left(K^{*}(s)\right)_{s} \backslash K^{* *}(s)$, and $n(x)=0$ otherwise. $\left(\left(K^{*}(s)\right)_{s}\right.$ is the set parallel to $K^{*}(s)$ at distance $s$, as defined below.) By Poincarés formula, the integral of $n(x)$ over the plane is $4 s\left|\partial K^{*}(s)\right|$, and one recovers (7).

Other conjugacy properties between $K^{*}(s)$ and $K^{* *}(s)$ are easily obtained: for instance, the sum of their widths in any direction is equal to $2 s$. Those properties, combined with $K \subseteq K^{* *}(s) \subseteq B\left(C_{e}, R(K)\right)$ and Theorem I, enable the obtention of straightforward lower and upper bounds for quantities associated with $K^{*}(s)$ and $K^{* *}(s)$, expressed in terms of quantities associated with $K$. We also mention in this context the following:

(p) $K^{* *}(s) \subseteq K^{*}(s)$ iff $s \geq d(K)$,

(q) $d\left(K^{* *}(s)\right)=d(K)$ if $s \geq d(K)$,

and its conjugate

(r) $\delta\left(K^{*}(s)\right)=2 s-d(K)$ if $s \geq d(K)$.

(A proof of these last statements will be given in $\S 4$.)

Let us now recall that $K$ being a plane convex set of boundary $\partial K$, the set parallel to $K$ at distance $t$, denoted by $K_{t}$, is defined as

$$
K_{t}:= \begin{cases}\bigcup_{x \in K} B(x, t)=\left\{x: \min _{y \in K}|x-y| \leq t\right\} & \text { for } t \geq 0, \\ \left\{x \in K: \max _{y \in \partial K}|x-y| \geq-t\right\} & \text { for } t \leq 0 .\end{cases}
$$

Obviously, $K_{t}$ is nonempty for $t \geq-r(K)$ only, where $r(K)$ is the inner radius of $K$. We have

(s) $K^{* *}(s)=\bigcap$ (all disks of radius $s$ containing $K$ )

$$
=\bigcap(\text { all } s \text {-overconvex sets containing } K) \text {. }
$$


(t) $\left.K^{*}(s)\right)_{s}=\bigcup($ all disks of radius $s$ containing $K$ )

$=\bigcup$ (all $s$-overconvex sets containing $K$ ).

Proof. (s) readily follows from (i) and (o). On the other hand, a point $y$ belongs to some disk $B(x, s)$ containing $K$ (that is, $\left.x \in K^{*}(s)\right)$ iff $|x-y| \leq s$, that is $y \in\left(K^{*}(s)\right)_{s}$.

The conjugacy symmetry, while apparently broken when comparing (s) with $(\mathrm{t})$, will be restored in $\S 4$ by replacing " $s$-overconvex sets containing $K$ " by "sets of constant width $s$ containing $K$."

We shall now compare the "growth" properties of adjoint sets with those of parallel sets.

Theorem II. Let $K$ be convex and $s \geq R(K)$. Then

$$
\begin{aligned}
& \left(K^{*}(s+t)\right)_{-t}=K^{*}(s)=\left(K_{t}\right)^{*}(s+t) \quad \text { for } t \geq 0, \\
& \left(K^{*}(s+t)\right)_{-t} \subseteq K^{*}(s) \subseteq\left(K_{t}\right)^{*}(s+t) \quad \text { for }-s \leq t \leq 0 .
\end{aligned}
$$

Proof. (i) Suppose $t \geq 0$. The following chain of equivalences holds: $x \in$ $K^{*}(s+t)_{-t} \Leftrightarrow B(x, t) \subseteq K^{*}(s+t) \Leftrightarrow \forall z \in K, B(x, t) \subseteq B(z, s+t) \Leftrightarrow \forall z \in K$, $|x-z| \leq s \Leftrightarrow x \in K^{*}(s)$.

Similarly, $x \in\left(K_{t}\right)^{*}(s+t) \Leftrightarrow K_{t} \subseteq B(x, s+t) \Leftrightarrow K \subseteq B(x, s) \Leftrightarrow x \in$ $K^{*}(s)$.

(ii) Assume $t \leq 0 . x \in K^{*}(s+t)_{-t} \Leftrightarrow \exists y \in K^{*}(s+t)$ (i.e. $\left.K \subseteq B(y, s+t)\right)$, with $x \in B(y,-t) \Rightarrow \forall z \in K,|x-z| \leq s \Leftrightarrow x \in K^{*}(s)$.

Similarly, $x \in K^{*}(s) \Leftrightarrow K \subseteq B(x, s) \Rightarrow K_{t} \subseteq B(x, s+t) \Leftrightarrow x \in\left(K_{t}\right)^{*}(s+t)$.

For fixed $s$, let us now consider the areas of the sets involved in (9) as a function of $t$, and define $f(t):=\left|\left(K^{*}(s)\right)_{t}\right|$ and $g(t):=\left|K^{*}(s+t)\right|$. One can show both functions to be $C^{2}$ in a neighborhood of $t=0$. Since $f(t) \leq g(t)$ with $f(0)=g(0)$, one has $f^{\prime}(0)=g^{\prime}(0)$ and $f^{\prime \prime}(0) \leq g^{\prime \prime}(0)$. On the other hand, Steiner formula (see e.g. [1]) gives $f^{\prime}(0)=\left|\partial K^{*}(s)\right|$ and $f^{\prime \prime}(0)=2 \pi$. We have as a result the following:

Theorem III. The perimeters $\left|\partial K^{*}(s)\right|,\left|\partial K^{* *}(s)\right|$ and areas $\left|K^{*}(s)\right|,\left|K^{* *}(s)\right|$ of a conjugate pair satisfy

$$
\begin{gathered}
\frac{d}{d s}\left|K^{*}(s)\right|=\left|\partial K^{*}(s)\right| . \\
\frac{d}{d s}\left|K^{* *}(s)\right|=s \frac{d}{d s}\left|\partial K^{* *}(s)\right| . \\
\frac{d}{d s}\left|\partial K^{*}(s)\right| \begin{cases}=2 \pi & \text { if } K \text { is } s \text {-overconvex } \\
>2 \pi & \text { otherwise. }\end{cases}
\end{gathered}
$$

(11) follows from (10) and Theorem I. We have also taken into account in (12) the fact that $\left|\partial K^{* *}(s)\right|$ is constant in $s$ iff $K$ is $s$-overconvex.

Remark. The lack of symmetry between (10) and (11) was to be expected, since by $(\mathrm{d})$ and $(\mathrm{j}) K^{*}(s)$ is increasing in $s$ whereas $K^{* *}(s)$ is decreasing in $s$. It is here that the concept of conjugacy breaks down.

\section{RELATION WITH THE SETS OF CONSTANT WIDTH}

Let us now address the question of characterising the sets $K$ left invariant by the adjoint transform, first raised by Eggleston in another context [10]. Before 
doing so, we shall recall that a convex set $K$ is said to be of constant width $d$ if its support function satisfies everywhere $h(\phi)+h(\pi+\phi)=d$. This amounts (in the euclidean case) to say that $K$ is complete, that is such that the addition of any point to $K$ strictly increases its diameter. (See Chakerian and Groemer [9] for a recent and fairly exhaustive survey of properties of sets of constant width.) Finally, a set $K_{c}$ is said to be a completion of $K$ if $K \subseteq K_{c}, d(K)=d\left(K_{c}\right)$ and $K_{c}$ is of constant width. Every convex body possesses at least one completion (see e.g. [6]). This incidentally will be proved in this section.

Suppose $K=\mathscr{A}(K, s)=K^{*}(s)$. Then, clearly, the diameter of $K$ must be equal to $s$. Suppose now a point $y \notin K$ is added to $K$. As $K=K^{*}(s)$, this imply $y$ to be at distance bigger than $s$ from some point of $K$, i.e. $K$ is complete. Conversely, let $K$ be of constant width $s$. By (f), one has $K \subseteq$ $K^{*}(s)$. Suppose there is a point $y$ belonging to $K^{*}(s)$ but not to $K$. The distance between $y$ and any point of $K$ is then less or equal to $s$, contradicting the fact that $K$ is complete. Therefore $K^{*}(s) \subseteq K$, and we have [10]:

(u) $K=\mathscr{A}(K, s)=K^{*}(s)$ iff $K$ is of constant width $s$.

The sets of constant width are therefore the fixed points of the adjoint transform, whereas the overconvex sets are the fixed points of the double adjoint transform. Clearly, a set of constant width $s$ is $s$-overconvex.

In the following, $s$ will be taken equal to the diameter $d=d(K)$ of $K$ and will be omitted. Let $K_{c}$ be a completion of $K$. As $K \subseteq K_{c}=\mathscr{A}\left(K_{c}, d\right)$, we have, using (e) and (k), the very useful corollary:

(v) Any completion $K_{c}$ of $K$ satisfies $K^{* *} \subseteq K_{c} \subseteq K^{*}$.

$(\mathrm{p}),(\mathrm{q})$ and (r) are direct consequences of (v). The following finite construction, we believe to be new, leads to the obtention of two completions of an arbitrary, plane body:

Construction. Given a convex body $K$ of diameter $d$, select two points $x_{1}$ and $x_{2}$ belonging to the boundary $\partial K$ of $K$ such that $\left|x_{1}-x_{2}\right|=d$. The line $L\left(x_{1}, x_{2}\right)$ passing through $x_{1}$ and $x_{2}$ divides the plane into two closed half planes $E_{+}$and $E_{-}$. We now construct two sets $K_{a}$ and $K_{b}$ as

$$
\begin{aligned}
& K_{a}:=\left(K^{*} \cap E_{+}\right) \cup\left(K^{* *} \cap E_{-}\right) . \\
& K_{b}:=\left(K^{*} \cap E_{-}\right) \cup\left(K^{* *} \cap E_{+}\right) .
\end{aligned}
$$

As announced, we have

(w) $K_{a}$ and $K_{b}$ are completions of $K$.

Proof. Choose a coordinate system in which the line $L\left(x_{1}, x_{2}\right)$ has direction $\phi=0$. Then the support functions of $K_{a}$ and $K_{b}$ are respectively given by:

$$
h_{a}(\phi)=\left\{\begin{array}{ll}
h^{*}(\phi), & 0 \leq \phi \leq \pi, \\
h^{* *}(\phi), & \pi \leq \phi \leq 2 \pi,
\end{array} \quad h_{b}(\phi)= \begin{cases}h^{* *}(\phi), & 0 \leq \phi \leq \pi, \\
h^{*}(\phi), & \pi \leq \phi \leq 2 \pi,\end{cases}\right.
$$

where $h^{*}$, resp. $h^{* *}$ are the support functions of $K^{*}$, resp. $K^{* *}$. By the choice of $x_{1}$ and $x_{2}$, we have $x_{i} \in K^{*}$, as well as $x_{i} \in K^{* *}$ for $i=1,2$, and so $K_{a}$ and $K_{b}$ are convex bodies possessing continuous support functions, which by (4) satisfy

$$
h_{a}(\phi)+h_{a}(\pi+\phi)=h_{b}(\phi)+h_{b}(\pi+\phi)=d
$$

which completes the proof. 


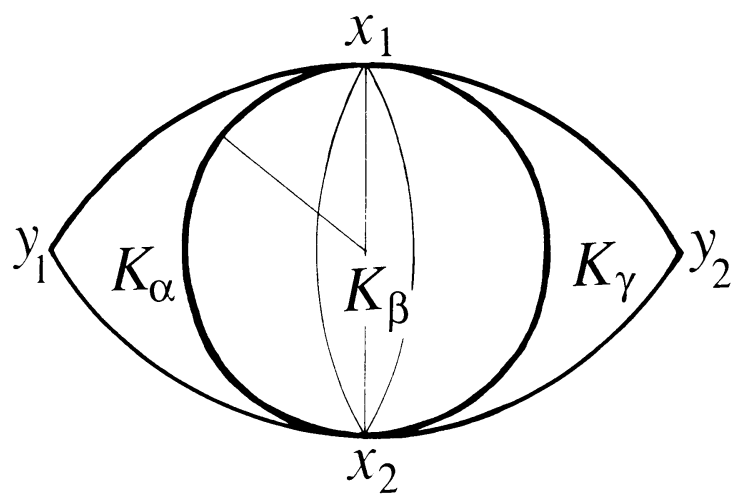

Figure 1

(w) contains as a corollary the theorem of Pàl, stating that every bounded set possesses at least one completion. Alternatively [12], one can observe that the set $\frac{1}{2}\left(K^{*}+K^{* *}\right)$ (Minkowski sum) possesses a support function satisfying (16) and, taking (f) and (g) into account, constitutes then a completion of $K$ as well.

By (v), the intersection of all completions $K_{c}$ of $K$ contains $K^{* *}$, and the union of all completions is contained in $K^{*}$. On the other hand, $K_{a} \cap K_{b}=K^{* *}$, and $K_{a} \cup K_{b}=K^{*}$. We therefore get the following new characterisation, to be compared with (s) and $(\mathrm{t})$ :

Theorem IV. Let $K$ be a plane compact set of diameter $d$. Then

$$
\begin{aligned}
K^{* *}(d) & =\bigcap(\text { all completions of } K) . \\
K^{*}(d) & =\bigcup(\text { all completions of } K) .
\end{aligned}
$$

Remark. Clearly, $K_{a}=K_{b}$ iff $K^{*}=K^{* *}$ is, in which case its completion is trivially unique. When however this is not the case, the set

$$
\left\{h_{\lambda}:=\lambda h_{a}+(1-\lambda) h_{b}\right\}_{\lambda \in[0,1]}
$$

constitutes an infinite one-parameter family of support functions of completions of $K$. One can easily convince oneself that the family under consideration is generally far from being exhaustive.

As an example, let $K$ be a segment of length $d$ and extremities $x_{1}$ and $x_{2}$ (see Figure 1). $K^{*}(d)$ is the lens of radius $d$ and extremities $y_{1}$ and $y_{2}$, whereas $K^{* *}(d)$ is the lens of radius $d$ and extremities $x_{1}$ and $x_{2}$. Both $K_{a}$ and $K_{b}$ are Reuleaux triangles with vertices $x_{1}, x_{2}, y_{1}$, respectively $x_{1}, x_{2}, y_{2}$.

As any set $\widetilde{K}$ of diameter $d$ contains a congruent copy of $K$, and as $\widetilde{K}^{*}(d) \subseteq$ $K^{*}(d)$ by (e), Theorem IV applied on $\widetilde{K}$ implies that any set of constant width can be contained in a congruent copy of $K^{*}(d)$, i.e. $K^{*}(d)$ is a universal cover. However, $K^{*}(d)$ does not constitute a minimal universal cover, i.e. it contains proper subsets themselves universal covers. To see this, consider $K^{* *}(d / 2)$, the circumcircle of $K$, whose boundary splits $K^{*}(d)$ into three disjoint parts 
$K_{\alpha}, K_{\beta}=K^{* *}(d / 2)$ and $K_{\gamma}$. Since it is impossible for any set of diameter $d$ to have a nonempty intersection with both $K_{\alpha}$ and $K_{\gamma}$, one then deduces (by reflection symmetry around the axis $x_{1} x_{2}$ ), that $K_{\alpha} \cup K_{\beta}$ (or equivalently $K_{\gamma} \cup K_{\beta}$ ) alone is a universal cover. Moreover, the set in question constitutes a minimal universal cover, since no proper subset of itself can contain at the same time the disk and the Reuleaux triangle of diameter $d$.

The identification of $K_{\alpha} \cup K_{\beta}$ as a minimal universal cover (and apparently the only one discovered so far) belongs to Eggleston [11]. Our proof is however considerably simpler than the original one.

\section{Application: Area of a Random Set of maXimal given diameter}

We consider in this section the following two stochastic processes:

$n$ points are sequentially (respectively simultaneously) thrown in the plane, uniformly with respect to the Lebesgue measure, submitted to the condition that the distance between any two points must not exceed a fixed distance $D$.

As we shall see, the probability measures generated by the two processes are different.

Let us begin with the sequential process: we denote by $x_{0}, x_{1}, \ldots, x_{n}$ the positions of the $n+1$ first points realised in the process, and by $K_{n}$ their convex hull. We fix $x_{0}=0$ for convenience. By construction, $d\left(K_{n}\right) \leq D$, and the next point $x_{n+1}$ will be uniformly distributed in the region $\left\{x: \max _{i=0, \ldots, n}\left|x-x_{i}\right| \leq\right.$ $D\}=K_{n}^{*}(D)$. As $K_{n+1}^{*}(D) \subseteq K_{n}^{*}(D)$, the limit $K_{\infty}^{*}(D):=\lim _{n \rightarrow \infty} K_{n}^{*}(D)$ exists for any realisation. The following theorem holds:

Theorem V. In the sequential process, $K_{\infty}^{*}(D)$ is a set of constant width $D$ with probability one.

Proof. Suppose the assertion does not hold; by (p) and (u), $K_{\infty}^{*}(D) \backslash K_{\infty}^{* *}(D)$ is then nonempty, and moreover (see e.g. (3), (7) and (p)) of strictly positive area. By construction, any point $x$ thrown in this set will "delete" a part of $K_{\infty}^{*}(D)$, because a further point, in order to satisfy the diameter constraint, will be confined in $\left(K_{\infty}^{*}(D) \cap B(x, D)\right) \subset K_{\infty}^{*}(D)$ only. This implies the survival probability of $K_{\infty}^{*}(D)$ after $m$ throws to be $\left(\left|K_{\infty}^{* *}(D)\right| /\left|K_{\infty}^{*}\right|(D)\right)^{m}$; hence $K_{\infty}^{* *}(D)=K_{\infty}^{*}(D)$ with probability one.

(See Sallee [14] for a deterministic version of Theorem V.)

Let $A\left(x_{1}, \ldots, x_{n}\right)$ be a sufficiently regular function of the points positions. Bearing in mind the fact that each point is conditionally distributed with respect to its predecessors, one finds that the average of $A$ is given by

$$
\left\langle A\left(x_{1}, \ldots, x_{n}\right)\right\rangle_{\text {seq }}=\int \frac{\theta\left(D-d\left(K_{n}\right)\right) A\left(x_{1}, \ldots, x_{n}\right) d x_{1} \cdots d x_{n}}{\left|K_{0}^{*}(D)\right| \cdots\left|K_{n-1}^{*}(D)\right|}
$$

where $\theta(\cdots)$ denotes the unit step function. In particular,

$$
a_{\infty, \text { seq }}:=\lim _{n \rightarrow \infty}\left\langle\left|K_{n}^{*}(D)\right|\right\rangle_{\text {seq }}
$$

exists, and as a consequence of Theorem V satisfies:

$$
\frac{\pi-\sqrt{3}}{2} D^{2}<a_{\infty, \text { seq }}<\frac{\pi D^{2}}{4} .
$$

The bounds in (21) correspond to the minimal, respectively maximal area of a set of constant width $D$, realised as it is well known by the Reuleaux triangle, 
respectively the disk. On the other hand, it is easy to realise that these two sets are themselves destroyed with nonzero probability for $n=3$ already, whence the strict inequalities in (21). Improving (21) seems to be awkward.

In contrast, the corresponding problem for the simultaneous process can be fully solved. Actually, using the same notation, we have the somewhat surprising theorem:

Theorem VI. In the simultaneous process, $K_{\infty}^{*}(D)$ is a disk radius $D / 2$ with probability one.

Theorem VI is equivalent to the statement

$$
\lim _{n \rightarrow \infty} a_{n, \operatorname{sim}}=\frac{\pi D^{2}}{4}
$$

where

$$
a_{n, \operatorname{sim}}:=\left\langle\left|K_{n}^{*}(D)\right|\right\rangle_{n, \operatorname{sim}} \cdot
$$

Let us make it clear that, by construction, the average of an observable $A\left(x_{1}, \ldots, x_{n}\right)$ is now given by

$$
\left\langle A\left(x_{1}, \ldots, x_{n}\right)\right\rangle_{n, \operatorname{sim}}:=\frac{\int \theta\left(D-d\left(K_{n}\right)\right) A\left(x_{1}, \ldots, x_{n}\right) d x_{1} \cdots d x_{n}}{\int \theta\left(D-d\left(K_{n}\right)\right) d x_{1} \cdots d x_{n}}
$$

where as before $K_{n}$ is the convex hull of $x_{0}=0, x_{1}, \ldots x_{n}$. It is now necessary to specify the index $n$ outside the bracket, for the average of, say, a quantity $A\left(x_{1}, x_{2}\right)$ depending on two points only will generally be sensitive to the number $n \geq 2$ of particles to be simultaneously thrown. Let us now consider:

$$
\mu_{n}:=\int \theta\left(D-d\left(K_{n}\right)\right) d x_{1} d x_{2} \cdots d x_{n} .
$$

Suppose $K_{n}$ satisfies $D \geq d\left(K_{n}\right)$. Then $D \geq d\left(K_{n+1}\right)$ iff $x_{n+1} \in K_{n}^{*}(D)$, and therefore

$$
a_{n, \operatorname{sim}}=\frac{\int \theta\left(D-d\left(K_{n}\right)\right)\left|K_{n}^{*}(D)\right| d x_{1} \cdots d x_{n}}{\int \theta\left(D-d\left(K_{n}\right)\right) d x_{1} \cdots d x_{n}}=\frac{\mu_{n+1}}{\mu_{n}} .
$$

Let $\bar{K}_{n}$ be the convex hull of the points $\bar{x}_{0}=0, \bar{x}_{1}, \ldots, \bar{x}_{n}$. We have

$$
\begin{gathered}
\int \theta\left(D-d\left(\left\{x_{n+1}\right\} \cup K_{n}\right)\right) \theta\left(D-d\left(\left\{\bar{x}_{n+1}\right\} \cup \bar{K}_{n}\right)\right) d x_{1} \\
\cdots d x_{n+1} d \bar{x}_{1} \cdots d \bar{x}_{n+1} \\
\leq \int \theta\left(D-d\left(K_{n}\right)\right) \theta\left(D-d\left(\left\{x_{n+1}\right\} \cup\left\{\bar{x}_{n+1}\right\} \cup \bar{K}_{n}\right)\right) d x_{1} \\
\cdots d x_{n+1} d \bar{x}_{1} \cdots d \bar{x}_{n+1} .
\end{gathered}
$$

The above inequality is an immediate consequence of the fact that the measure of the positions available to $x_{n+1}$ (conditionally to $d\left(K_{n}\right) \leq D, d\left(\bar{K}_{n}\right) \leq D$ and $\left.\bar{x}_{n+1} \in\left(\bar{K}_{n}\right)^{*}(D)\right)$ is on average bigger in the 1.h.s. than in the r.h.s. (observe that $K_{n}$ and $\bar{K}_{n}$ are generated in a similar way). But equation (27) is the same as

$$
\mu_{n+1}^{2} \geq \mu_{n} \mu_{n+2}
$$


and therefore, by (26), $a_{n+1}$, sim $\leq a_{n}$, sim , which was intuitively to be expected. This proves the existence of the limit $a_{\infty}$, sim $:=\lim _{n \rightarrow \infty} a_{n, \text { sim }}$, which can as before be shown to satisfy $a_{\infty, \operatorname{sim}} \leq \pi D^{2} / 4$. On the other hand,

$$
\mu_{n} \geq \int_{(B(0, D / 2))^{n}} \theta\left(D-d\left(K_{n}\right)\right) d x_{1} \cdots d x_{n}=\left(\frac{\pi D^{2}}{4}\right)^{n}
$$

Hence, from (26),

$$
a_{\infty, \operatorname{sim}}=\lim _{n \rightarrow \infty}\left(\mu_{n}\right)^{1 / n} \geq \pi D^{2} / 4
$$

and the theorem is proved.

Remark. Believe it or not, in statistical mechanics $\mu_{n}$ is called microcanonical partition function and Theorem VI is equivalent to: "The Gibbs free energy of an assembly of $N$ particles in the plane with Hamiltonian $H\left(x_{1}, \ldots, x_{n}\right)=$ $\lambda \max _{i<j}\left|x_{i}-x_{j}\right|^{2}$ at temperature $\beta^{-1}$ is $g=\beta^{-1} \ln (4 \beta \lambda / \pi)$; moreover, the shape of their convex hull is asymptotically circular with radius $(N / 4 \beta \lambda)^{-1 / 2}$." We refer the reader to $[2,3]$ for further details.

\section{ACKNOWLEDGMENT}

I would like to thank B. Tóth for useful discussion about $\S 5$.

\section{REFERENCES}

1. C. Bandle, Isoperimetric inequalities and applications, Pitman, 1980.

2. F. Bavaud, Statistical mechanics of convex bodies, J. Stat. Phys. 57 (1989), 1059-1068.

3. __ Isoperimetric phase transitions of two-dimensional droplets, Comm. Math. Phys. (to appear).

4. E. Blanc, Les ensembles surconvexes plans, Ann. Sci. École Norm. Sup. Pisa 60 (1943), 215-246.

5. _ـ Sur une généralisation des domaines d'épaisseur constante, C. R. Acad. Sci. Paris 219 (1944), 662-663.

6. T. Bonnesen and W. Fenchel, Theorie der Konvexen Körper, Chelsea, 1948.

7. H. Bückner, Über Flächen von fester Breite, Jber. Deutsch. Math.-Verein. 46 (1936), 96-139.

8. N. Cameron, Introduction to linear and convex programming, Cambridge Univ. Press, 1985.

9. G. D. Chakerian and H. Groemer, Convex bodies of constant width, Convexity and its Applications, Birkhäuser, 1983.

10. H. G. Eggleston, Sets of constant width in finite dimensional Banach spaces, Israel J. Math. 3 (1965), 163-172.

11. _ Convexity, Cambridge Univ. Press, 1969.

12. H. Maehara, Convex bodies forming pairs of constant width, J. Geom. 22 (1984), 101-107.

13. A. E. Mayer, Eine Überkonvexität, Math. Z. 39 (1934), 511-531.

14. G. T. Sallee, Reuleaux polytopes, Mathematika 17 (1970), 315-323.

15. _ _ Pairs of sets of constant relative width, J. Geom. 29 (1987), 1-11.

16. L. A. Santaló, Integral geometry and geometric probability, Addison-Wesley, 1976.

Department of Mathematics, Heriot-Watt University, EH14 4AS Edinburgh, United KINGDOM UK-SCOTLAND

Current address: Institut de Mathématiques Appliquées, Université de Lausanne, BFSH2, CH-

1015 Lausanne, Switzerland

E-mail address: fbavaud@ulys.unil.ch 